Published in final edited form as:

Dig Dis Sci. 2016 September ; 61(9): 2741-2748. doi:10.1007/s10620-016-4181-7.

\title{
Severe Acute Hepatocellular Injury Attributed to OxyELITE Pro: A Case Series
}

\author{
Lauren A Heidemann, M.D. ${ }^{1}$, Victor J Navarro, M.D. ${ }^{2}$, Jawad Ahmad, M.D. ${ }^{3}$, Paul H. Hayashi, \\ M.D. ${ }^{4}$, Andrew Stolz, M.D. ${ }^{5}$, David E. Kleiner, M.D. ${ }^{6}$, and Robert J. Fontana, M.D. ${ }^{1}$ on behalf \\ of the DILIN Investigators \\ Lauren A Heidemann: Iheidema@med.umich.edu; Victor J Navarro: NavarroV@einstein.edu; Jawad Ahmad: \\ jawad.ahmad@mountsinai.org; Paul H. Hayashi: paul_hayashi@med.unc.edu; Andrew Stolz: astolz@usc.edu; David E. \\ Kleiner: kleiner@mail.nih.gov \\ ${ }^{1}$ Department of Internal Medicine, University of Michigan Medical Center, Ann Arbor, MI \\ 2Department of Medicine, Einstein Healthcare Network, Philadelphia, PA \\ ${ }^{3}$ Department of Medicine, Icahn School of Medicine at Mount Sinai Medical Center, New York, NY \\ ${ }^{4}$ University of North Carolina, Chapel Hill, NC \\ ${ }^{5}$ University of Southern California, Los Angeles, CA \\ ${ }^{6}$ Laboratory of Pathology, National Cancer Institute, National Institutes of Health, Bethesda, MD
}

\section{Abstract}

BACKGROUND/AIMS-Herbal and dietary supplement (HDS) hepatotoxicity is increasingly being reported in the United States. This case series describes the presenting clinical features and outcomes of 7 patients with liver injury attributed to OxyELITE Pro enrolled in the Drug Induced Liver Injury Network (DILIN) study.

METHODS-The 6 month outcomes of patients with hepatotoxicity attributed to OxyELITE Pro enrolled in the DILIN prospective registry between 2004 and 2015 are presented.

RESULTS-Six of the 7 patients (86\%) presented in 2013 with symptoms of hepatitis and acute hepatocellular injury. The median duration of OxyELITE Pro use was 18 weeks (range: 5 to 102 weeks). Median age was 36 years (range: 28 to 62 ), $86 \%$ were female, and $43 \%$ were Asian. One patient had rash, none had eosinophilia and 3 had antinuclear antibody reactivity. The median peak ALT was $2242 \mathrm{U} / \mathrm{L}$, alkaline phosphatase $284 \mathrm{U} / \mathrm{L}$ and bilirubin $15.0 \mathrm{mg} / \mathrm{dL}$. Six patients (86\%) were hospitalized, 3 developed acute liver failure and 2 underwent liver transplantation. DILIN causality scores for OxyELITE Pro were definite in 1, highly likely in 3, probable in 2, and possible in 1 . Four of the 5 patients without liver transplant recovered completely within 6 months while one patient had mild residual ALT elevations.

Address all correspondence to: Robert J. Fontana, MD, Professor of Internal Medicine, 3912 Taubman Center, Ann Arbor, MI 48109-0362, Tel: (734)-936-4780, Fax: (734)-936-7392, rfontana@med.umich.edu.

Author contributions: All authors contributed to the collection of clinical data, data analysis, and initial and final drafting of the manuscript. DEK provided expert review of the available liver histopathology.

Conflict of interest disclosure: No conflicts of interest to disclose. 
CONCLUSIONS-Seven cases of severe acute hepatocellular injury attributed to OxyELITE Pro are reported. These results reinforce the need to assess for HDS supplement use in patients presenting with unexplained acute hepatitis and point to the need for additional regulatory oversight of HDS products.

\section{Keywords}

hepatotoxicity; herbal and dietary supplement; drug-induced liver injury

\section{Introduction}

An estimated 50 to $60 \%$ of adult Americans ingest an herbal or dietary supplement (HDS) on a regular basis for a variety of touted health benefits $(1,2)$. Although HDS products are perceived to be safe and "natural", they do not undergo routine efficacy or safety testing. A recent study reported that at least 23,000 emergency department visits in the United States each year are attributed to adverse events from dietary supplements (3). Despite this, the Food and Drug Administration (FDA) has limited authority to regulate the manufacturing and content of HDS products (4).

OxyELITE Pro (USP Labs, Dallas, TX) is a multi-ingredient nutritional supplement used by many individuals as a weight-loss and energy-enhancing aid (5). In mid to late 2013, over 40 cases of severe acute hepatitis and liver failure were linked to the use of the "Super Thermo" formulation of OxyELITE Pro, primarily amongst individuals residing in Hawaii (5-8). The etiology and risk factors for hepatotoxicity amongst these patients remain under investigation. However, the sudden outbreak followed shortly after a major modification of the components of OxyELITE Pro with the addition of aegeline, a constituent of the native Ayurvedic herbal product made from the bark of the bael tree (Aele marmelos). The product added to OxyELITE Pro, however, had been synthesized by a manufacturer in China and its purity, safety and relationship to the natural herbal product was not clear.

Before, during and after the outbreak of liver injury attributed to OxyELITE Pro, the Drug Induced Liver Injury Network (DILIN) had been conducting a prospective multicenter study of the etiologies and outcomes of patients who developed liver injury due to ingestion of drugs and HDS products in the United States $(9,10)$. Over the course of DILIN study, the proportion of cases attributed to various HDS products including body building agents and weight loss products increased markedly, from 7\% in 2004-2006 to 20\% since 2012 (11). Among the 246 adjudicated cases of DILI attributed to HDS products, 7 were considered to be due to OxyELITE Pro, 6 of which presented during mid-to-late 2013. The aim of the current study was to describe the presenting features, clinical course and outcomes of 7 patients enrolled in the DILIN prospective study with liver injury attributed to OxyELITE Pro. 


\section{Methods}

\section{DILIN prospective study}

The protocol for this multicenter observational study was approved by the Institutional Review Boards at each clinical site and all enrolled subjects provided written informed consent. Liver injury onset was defined as the first date after a subject taking any medication or HDS product met the predefined laboratory criteria for study entry. Specifically, all subjects had to meet one of the following laboratory criteria on 2 consecutive blood draws: (1) a serum aspartate aminotransferase (AST) or alanine aminotransferase (ALT) level that exceeded 5 times the upper limit of normal (ULN) (or 5 times a pretreatment baseline value if abnormal); (2) a serum alkaline phosphatase (Alk P) that exceeded 2 times the ULN (or 2 times the pretreatment value if abnormal); or (3) a total bilirubin of $2.5 \mathrm{mg} / \mathrm{dL}$ or greater, or an international normalized ratio (INR) greater than 1.5 accompanied by an enzyme elevation. To qualify for enrollment, study participants had to be enrolled within 6 months of onset.

A detailed medical history was obtained at the enrollment study visit and medical records were retrieved and relevant information was extracted. Additional laboratory and radiological testing were performed if necessary to more fully characterize the event and exclude competing etiologies. Specifically, testing for hepatitis A, B, C, HIV, autoantibodies, $\mathrm{CMV}$, and EBV infection was obtained in all subjects and serum was stored in a sample repository for future diagnostic testing and ancillary studies. In this regard, available samples were later tested for IgG anti-HEV and, if positive, for IgM anti-HEV as previously reported (12). All enrolled patients were asked to return for a follow-up visit at 6 months after enrollment, and those with persistent evidence of liver injury at 6 months after onset were then asked to return at 12 and 24 months.

The causal relationship between the liver injury episode and the implicated agent(s) was evaluated in a standardized fashion by the DILIN Causality Committee (13). A DILIN expert opinion causality score varying from 1 (definite: $295 \%$ likelihood), 2 (highly likely: 75\%-94\% likelihood), 3 (probable: 50\%-74\% likelihood), 4 (possible: $25 \%-49 \%$ likelihood) to 5 (unlikely: < $25 \%$ likelihood) was assigned by consensus agreement of committee members. In addition, an updated causality score using the standardized Roussel Uclaf Causality Assessment Method (RUCAM) was calculated for each case and implicated agent by manuscript authors in 2015 (14). By convention, RUCAM scores are grouped into likelihood levels as "excluded" ( $\leq 0)$, "unlikely" (1-2), "possible" (3-5), "probable" (6-8) and "highly probable" (> 8). In subjects with 2 or more implicated drugs or HDS products, an overall causality score was assigned to the case and then a causality score was also determined for each individual suspect drug or HDS product.

The pattern of liver injury was categorized based upon the R-ratio which was defined by the formula: $[\mathrm{ALT} / \mathrm{ULN}] \div[$ Alk P/ULN]. Cases were considered hepatocellular if $\mathrm{R}>5$, cholestatic if $\mathrm{R}<2$ and mixed if $\mathrm{R} 2-5$. The severity of the DILI episode was categorized on a 5-point scale from mild (1), moderate (2), moderate-hospitalized (3), severe (4), and fatal (5), where a fatal score was assigned only if the patient died or underwent liver transplantation due to DILI within 6 months of onset (8). 


\section{Liver histopathology}

Liver biopsy was not required and was not a component of the DILIN Prospective Study protocol; but if a biopsy were done as a part of clinical evaluation and care, a request was made for slides to be forwarded to the Laboratory of Pathology, NIH. All available specimens were reviewed by a single expert liver histopathologist (DEK) and scored for multiple histological features as well as an overall pattern of liver injury as previously described (15).

\section{Data collection and statistics}

Electronic case report forms (eCRFs) via InForm ${ }^{\mathrm{TM}}$ (Oracle Health Solutions, CA) were used to capture data in the DILIN prospective study. Data reported here were based on the data download from InForm ${ }^{\mathrm{TM}}$ in December 2015. Summary statistics were reported as median with range for continuous data and frequency with percentage for categorical data. SAS 9.4 [SAS Institute, Cary, NC] was used for all statistical analyses.

\section{Results}

Between 2004 and 2015, 1596 patients were enrolled in the DILIN prospective study, 7 of whom $(0.5 \%)$ had liver injury attributed to OxyELITE Pro. One case had injury onset in 2011 and the remaining 6 occurred between May and December 2013. These 7 cases represented $2.8 \%$ of the 246 adjudicated cases attributed to HDS products and $5.3 \%$ of the cases enrolled during calendar year 2013.

The demographic, clinical and laboratory features of the 7 patients with liver injury attributed to OxyELITE Pro are summarized in Table 1. All 7 patients were adults, the median age was 36 years (range 28-62) and $6(86 \%)$ were women. Self-reported race and ethnicity indicated that 4 were Caucasian (one being Hispanic) and 3 Asian. While the most common reason for taking OxyELITE Pro was weight loss, most patients had a normal body mass index, 1 being mildly overweight and only 1 obese. The median duration of OxyELITE Pro use before liver injury onset was 18 weeks, but ranged from 5 to 102 weeks. Two patients reported taking OxyELITE Pro in the past (before 2013) without ill effects and 1 patient took the product for 2 years but presented with liver injury 6 months after March 2013 when the product was modified. Only 1 patient was a moderate drinker and none had a history of alcohol abuse or obvious risk factors for viral hepatitis.

On clinical presentation, the pattern of serum enzyme elevations was hepatocellular in all patients; the median R ratio being 16 (range 11-107). Initial ALT values were above 1,000 $\mathrm{U} / \mathrm{L}$ in 5 patients and averaged $2950 \mathrm{U} / \mathrm{L}$. Initial Alk $\mathrm{P}$ values in contrast were typically normal $(\mathrm{n}=2)$ or only modestly elevated $(\mathrm{n}=5)$ in the range of $1.5-3$ times ULN. Serum total bilirubin values were raised in 6 patients and ranged widely $(0.4$ to $24.5 \mathrm{mg} / \mathrm{dL})$ and averaged $10.9 \mathrm{mg} / \mathrm{dL}$. Immunoallergic features were not prominent; 1 patient had rash, 2 had fever, and none had documented peripheral eosinophilia. Autoantibodies were present in 4 patients but none had other features of autoimmune hepatitis and corticosteroids were used in only 1 patient, who had rapidly progressive hepatic failure. Testing for acute HAV, HBV and $\mathrm{HCV}$ infection was negative in all patients, although one (case \#7) appeared to be a 
chronic carrier of hepatitis B who had a superimposed severe acute liver injury (having HBsAg without IgM anti-HBc and no detectable HBV DNA). Tests for acute HEV infection were available on 4 patients and were negative in all. Liver imaging was performed in all patients and none had evidence of biliary obstruction or hepatic masses.

\section{Causality assessment}

Formal causality assessment judged 1 case to be definite, 2 highly likely, 3 probable, and 1 possible liver injury due to exposure to OxyELITE Pro. (Table 1). Similarly, the RUCAM scores varied from 2 to 7, being in the range of unlikely in 1, possible in 4 and probable in 2 (calculations are shown in Supplementary Table 1). Five patients were taking other prescription medications or HDS products that were listed as implicated, but only in case \#1 were these agents considered a more probable cause of the hepatic injury.

\section{Clinical severity and outcomes}

The DILIN severity scores included 1 mild, 3 moderate-hospitalized, 1 severe and 2 fatal, the latter two undergoing emergency liver transplantation 24 days after clinical presentation. Liver histology was available for central review in 3 patients. One patient (Case \#2) with a percutaneous liver biopsy done 4 days after presentation and at the peak of injury showed a cholestatic hepatitis pattern best characterized as acute hepatitis with moderate, predominantly lobular inflammation, mild canalicular cholestasis, and scattered eosinophils (Figure 1A/1B). Recuts from a single paraffin block from each of the two explants were also available for review (Figure 1C/1D). One case showed submassive necrosis with few residual viable hepatocytes while the other showed necrosis in zone 1 and 3 with extensive bridging necrosis and early regenerative nodule formation. Although plasma cells were noted within the infiltrate at transplantation, neither had other features suggestive of autoimmune hepatitis. The residual parenchyma in both cases showed prominent cholestasis.

At last follow-up, at 22 and 26 months post-transplant, the 2 patients who underwent liver transplantation were doing well and had normal serum bilirubin and liver enzymes. Four of the other 5 patients had self-limited hepatitis with full recovery without corticosteroid or other specific therapy at month 6 but 1 patient (Case \#4) had evidence of mild biochemical liver injury at follow-up month 8 .

\section{Discussion}

These seven generally healthy, middle aged adults developed acute hepatitis after ingesting a widely used weight loss, multi-ingredient nutritional product called OxyELITE Pro for as short as 5 weeks to as long as 2 years. Outcomes were generally severe with 6 patients jaundiced and hospitalized, 3 developing acute liver failure, and 2 requiring emergency liver transplantation. Six of the 7 patients had taken OxyELITE Pro in mid-to-late 2013 which was the period that the reformulated Super Thermo formulation of OxyELITE Pro had been marketed and distributed. In all cases, the daily dose of OxyELITE Pro ingested was within the recommendations from the manufacturer. Interestingly, 3 patients had taken other OxyELITE Pro formulations without adverse consequences in the past, prior to 2013. Indeed, the long latency to onset in 2 patients (Case \#4 and \#5) may have been the result of 
taking OxyELITE Pro produced before 2013, when the constituents did not include aegeline and switching to the reformulated product in 2013. Evaluation for competing causes of liver injury was unrevealing in all except Case \#7 who presumably had pre-existing chronic HBV infection.

The histological pattern of liver injury from 4 cases was compatible with other reports of toxin or drug mediated liver injury. Submassive hepatic necrosis is usually due to drugs and or ischemia. In these cases, the pattern of necrosis and inflammation was not compatible with an ischemic etiology. In contrast to cases of acute liver failure ascribed to autoimmune hepatitis, there were no lymphoid aggregates and the infiltrate was not rich in plasma cells (16). Similarly, a mixed pattern of hepatitis and cholestatic features as noted in Case \#1 is typical of drug hepatotoxicity and is not characteristic of autoimmune hepatitis $(15,17)$. While 4 of the patients described here had detectable autoantibodies at the onset of illness, liver histology was generally not consistent with autoimmune hepatitis. In addition, none had evidence of hypergammaglobulinemia at presentation nor during follow-up, and 4 of the 5 who survived without liver transplantation had normal liver enzymes in follow-up and were never treated with corticosteroids. Thus, the presence of low and moderate autoantibody reactivity in these patients was probably due to the severity of the acute liver injury rather than as an indication of autoimmune liver disease.

OxyELITE Pro is a product that contains a number of vitamins, herbs, and other micronutrients and compounds. The Super Thermo OxyELITE Pro product was reformulated in April 2013, at the request of the US FDA, to remove the ingredient 1,3dimethylamylamine (DMAA), which had been linked to cardiovascular toxicity $(8,18)$. Chemical analysis of the Super Thermo formulation of OxyELITE Pro linked to cases of hepatotoxicity has demonstrated the presence of aegeline (N-(2-hydroxy-2(4methoxyphenyl)ethyl)-3-phenyl-2-propenamide). This compound can be extracted from Aegle marmelos (bael leafs) which has a long history of use in Ayurvedic medicine to improve "energy levels". It has been alleged that the aegeline in the Super Thermo formulation of OxyELITE Pro was chemically synthesized, and the possibility exists that the product included metabolic intermediates or was a racemic mixture with one isomer having hepatotoxic potential (19). Although rare published reports have suggested occasional adverse effects from aegeline consumption, the mechanism by which aegeline and/or other components of OxyELITE Pro might mediate liver damage is unknown. OxyELITE Pro also contains green tea extract,which has been linked to recent reports of severe hepatotoxicity in humans $(20,21)$. However, the amount of green tea extract is limited and there was no apparent relationship between the daily dose of OxyELITE Pro taken and severity of liver injury, either in this series or in those reported by the CDC or by observers in Honolulu. Nonetheless, recent weight loss could lower the threshold for inadvertent liver toxicity from catechins in green tea extract as was recently demonstrated in animal studies $(4,22,23)$.

The US FDA enjoined USP Labs to cease marketing and distribution of the SuperThermo formulation of OxyELITE Pro in October 2013 because it contained a new dietary ingredient, aegeline, for which the manufacturer had not provided evidence of safety, and this formulation was withdrawn from the marketplace shortly thereafter. 
When comparing our cases with the published information on the 29 cases from Hawaii in 2013, several common features are obvious (7). Firstly, the median age of the patients was similar (36 vs 33 years) and there was an over-representation of individuals of east Asian descent compared to the general US population ( $43 \%$ and $80 \%$ vs $5.5 \%$ ) raising the possibility of a genetic predilection to hepatotoxicity from this HDS product, as has been reported with other drugs $(24,25)$. In addition, most patients presented with severe acute hepatocellular injury after several months of OxyELITE Pro ingestion and all but one patient in the current series presented between April and November 2013 (Table 1). However, all of the DILIN cases occurred in the continental US in comparison to the larger outbreak that was reported primarily in Hawaii. An investigation of the Hawaii cases failed to demonstrate a common batch or expiration date of the OxyELITE Pro product that was consumed (26). The DILIN cases tended to have more severe outcomes, including a higher rate of hospitalization ( $86 \%$ vs. $38 \%$ ) and liver transplant or death (29\% vs. $10 \%)$, which may relate to ascertainment and/or referral bias. Autoimmune features were present in both series, although none from the current study had prominent autoimmune features.

Limitations of the current study include the small number of cases limiting the ability to identify risk factors for liver injury. In addition, we were not able to obtain a sample of the actual OxyELITE Pro formulation ingested for formal chemical analysis of potential hepatotoxic ingredients or adulterants as have been found in other HDS products (27). Likewise, we could not confirm that the patients were taking the Super Thermo formulation, although the timing was suggestive in 6 of the 7 cases. Another potential confounding factor was that several of the patients were taking other HDS products that have been associated with hepatotoxicity (28-30) but only in Case \#1 were these other products thought to be even probably related.

The outbreak of severe acute hepatitis due to OxyELITE Pro was striking and led quickly to the identification of the responsible agent. At the same time, there has been a gradually increasing rate of liver injury from HDS products reported largely due to multi-ingredient products similar to OxyELITE Pro that contain multiple vitamins, minerals, nutritional elements and herbal products or synthetic compounds. The components of the typical multiingredient dietary supplements often change and their purity and reliability are not well documented. These findings stress the importance of considering HDS products in assessing acute liver injury of unknown cause, reporting such incidents (to Medwatch if possible) and attempting to obtain the suspect product for chemical analysis. These findings also indicate a need for enhanced regulatory actions in monitoring the commercial availability of these widely used weight loss products.

\section{Supplementary Material}

Refer to Web version on PubMed Central for supplementary material.

\section{Acknowledgments}

Funding source: The Drug Induced Liver Injury Network (DILIN) is structured as an U01 cooperative agreement supported by the National Institute of Diabetes and Digestive and Kidney Diseases (NIDDK) of the National Institutes of Health $(\mathrm{NIH})$ with funds provided by the following grants: U01DK065211 (Indiana University 
[Indianapolis]), U01DK065184 (University of Michigan [Ann Arbor]), U01DK065201 (University of North Carolina [Chapel Hill], Asheville, Wake Forest Baptist Medical Center), U01DK083020 (University of Southern California, University of California-Los Angeles [Pfleger Liver Institute]), U01DK083027 (Albert Einstein Medical Center), U01DK100928 (Icahn School of Medicine at Mount Sinai), U01DK065176 (Duke Clinical Research Institute). Additional support was provided by the Intramural Division of the National Cancer Institute (NCI), NIH.

\section{DILIN Clinical Sites:}

Indiana University: Naga Chalasani, MD, PI; Marwan S. Ghabril, MD, Sub-I; Raj Vuppalanchi, MD, Sub-I; [Audrey Corne, RN, EdD, Study Coord; Sherrie Cummings, RN, BSN, Study Coord; Wendy Morlan, RN, Study Coord];

University of Michigan-Ann Arbor: Robert J. Fontana, MD, PI; Hari Conjeevaram, MD, Sub-I; Frank DiPaola, MD, Sub-I; [Kristin Chesney, MBA, Study Coord; Sophana Mao, Study Coord; Cassandra Coffman, Study Coord];

University of North Carolina-Chapel Hill: Paul Watkins, MD, PI; Jama Darling, MD, Sub-I; Paul H. Hayashi, MD, Sub-I; Steven Lichtman, MD, Sub-I; Steven Zacks, MD, MPH, Sub-I; [Tracy Russell, CCRP, Study Coord; Beth Madden-Embleton, Co-Coord]; Satellite Sites: Asheville: William Harlan, MD, PI; [Tracy Russell, CCRP, Study Coord]; Wake Forest Baptist Medical Center: Herbert Bonkovsky, MD, PI; [Denise Faust, Study Coord].

University of Southern California: Andrew Stolz, MD, PI; Neil Kaplowitz, MD, Sub-I; [Susan Milstein, RN, BSN, Study Coord]; Satellite Sites: University of California-Los Angeles (Pfleger Liver Institute): Francisco A. Durazo, MD, PI; [Yolanda Melgoza, Study Coord; Val Peacock, RN, BSN, Co-Coord];

Albert Einstein Medical Center: Victor J. Navarro, MD, PI; Simona Rossi, MD, Sub-I; [Maricruz Vega, MPH, Study Coord; Manisha Verma, MD, MPH, Study Coord];

Icahn School of Medicine at Mount Sinai: Joseph Odin, MD, PhD, PI; Jawad Ahmad, MD, Co-I; Nancy Bach, Sub-I; Meena Bansal, MD, Sub-I; Charissa Chang, MD, Sub-I; Douglas Dieterich, MD, Sub-I; Priya Grewal, MD, Sub-I; Lawrence Liu, MD, Sub-I; Thomas Schiano, MD, Sub-I; [Sherif Mikhail, MD, Study Coord; Monica Taveras, Study Coord];

DILIN Data Coordinating Center at Duke Clinical Research Institute: Huiman X. Barnhart, PhD, PI; David Goldstein, PhD, Sub-I; Katherine Galan, RN, Project Lead; Alex Hammett, Lead CCRA; Cathy Wickward, CRA; Kenari Marks, CTA; Michelle McClanahan-Crowder, Data Management; Carmel Puglisi-Scharenbroich, Data Management; Hoss Rostami, Data Management; Qinghong Yang, Programmer-Statistics; Jiezhun (Sherry) Gu, $\mathrm{PhD}$, Statistician; Tuan Chau, Lead Safety Associate;

National Institute of Diabetes and Digestive and Kidney Diseases (NIDDK): José Serrano, MD, Project Scientist; Rebecca J. Torrance, RN, MS, Clinical Trials Specialist; Rebekah Van Raaphorst, MPH, LT, USPHS, Health Research Administrator; Francisco O. Calvo, PhD, COC Contact; Jose Serrano, MD, PhD (Program Officer);Jay H. Hoofnagle, MD, Scientific Advisor; Averell H. Sherker, MD, FRCP(C), Program Officer.

\section{Abbreviations}
ALT
Alanine aminotransferase
ANA
Antinuclear antibody
Alk $\mathbf{P} \quad$ Alkaline phosphatase
AST Aspartate aminotransferase
DILI Drug induced liver injury
DILIN Drug Induced Liver Injury Network
HDS Herbal and dietary supplement
INR International normalized ratio
RUCAM Roussel Uclaf Causality Assessment Method 
ULN Upper limit of normal

\section{References}

1. Timbo BB, Ross MP, McCarthy PV, Lin CJ. Dietary Supplements in a National Survey: Prevalence of Use and Reports of Adverse Events. Journal of American Dietetic Association. 2006; 106:19661974.

2. Gahache J, Bailey R, Burt V, et al. Dietary supplement use among U.S. adults has increased since NHANES II (1988-1994). NCHS Data Brief. Center for Disease control and Prevention. 2011; 61:1-8.

3. Geller AI, Shehab N, Weidle NJ, et al. Emergency Department Visits for Adverse Events Related to Dietary Supplements. N Engl J Med. 2015; 373:1531-1540. [PubMed: 26465986]

4. Zheng EX, Navarro VJ. Liver Injury from Herbal, Dietary, and weight Loss Supplements: A review. J Clin Trans Hepatology. 2015; 3:93-98.

5. OxyELITE Pro Supplements Recalled, FDA Consumer Health Information [Internet]. U.S Food and Drug Administration. 2013 Nov 18. Available from: www.fda.gov/forconsumers/consumerupdates/ ucm374742.htm

6. Roytman M, Poerzgen P, Lee CL, et al. Outbreak of severe hepatitis linked to weight loss supplement OxyELITE Pro. Am J Gastroenterol. 2014; 109:1296-1298. [PubMed: 25091255]

7. Centers for Disease Control and Prevention. Acute hepatitis and liver failure following the use of a dietary supplement intended for weight loss or muscle building. MMWR. 2013; 62:816-818.

8. Foley S, Butlin E, Shields W, Lacey B. Experience with OxyELITE Pro and acute liver injury in active duty service members. Dig Dis Sci. 2014; 59:3117-3121. [PubMed: 24916713]

9. Fontana RJ, Watkins PB, Bonkovsky HL, et al. Drug-Induced Liver Injury Network (DILIN) Prospective Study. Drug Safety. 2009; 32:55-68. [PubMed: 19132805]

10. Fontana RJ, Hayashi PH, Gu J, et al. Idiosyncratic Drug-Induced liver Injury is associated with substantial morbidity and mortality within 6 months from onset. Gastroenterology. 2014; 147:96108. [PubMed: 24681128]

11. Navarro VJ, Barnhart H, Bonkovsky HL, et al. Liver injury from herbals and dietary supplements in the U.S. Drug Induced Liver Injury Network. Hepatology. 2014; 60:1399-1408. [PubMed: 25043597]

12. Davern TJ, Chalasani N, Fontana RJ, et al. Acute hepatitis E infection accounts for some cases of suspected drug induced liver injury. Gastroenterology. 2011; 141:1665-1672. [PubMed: 21855518]

13. Rockey DC, Seeff LB, Rochon J, et al. Causality assessment in Drug-induced liver injury using a structured expert opinion process: comparison to the Roussel-Uclaf causality assessment method. Hepatology. 2010; 51:2117-2126. [PubMed: 20512999]

14. Benichou C, Danan G, Flahault A. Causality assessment of adverse reaction to Drugs: an original model for validation of drug causality assessment methods: case reports with positive rechallenge. J Clin Epidemiol. 1993; 46:1331-1336. [PubMed: 8229111]

15. Kleiner DE, Chalasani NP, Lee WM, et al. Hepatic histological findings in suspected drug-induced liver injury: systematic evaluation and clinical associations. Hepatology. 2014; 59:661-670. [PubMed: 24037963]

16. Stravitz RT, Lefkowitch JH, Fontana RJ, et al. Autoimmune acute liver failure: Proposed clinical and histological criteria. Hepatology. 2011; 53:517-526. [PubMed: 21274872]

17. Suzuki A, Brunt EM, Kleiner DE, et al. The use of liver biopsy evaluation in discrimination of idiopathic autoimmune hepatitis versus drug-induced liver injury. Hepatology. 2011; 54:931-939. [PubMed: 21674554]

18. Lammie J. Editor and safety Panel lead. Report of the Department of Defense 1,3dimethylamylamine (DMAA) safety review and panel. 2013:97-98.

19. FDA takes action to protect consumers from potentially dangerous supplements. FDA News release. 2015 Nov 17. at http://www.fda.gov/NewsEvents/Newsroom/PressAnnouncements/ ucm473099.htm 
20. Navarro VJ, Bonkovsky HL, Hwang SI, Vega M, Barnhart H, Serrano J. Catechins in dietary supplements and hepatotoxicity. Dig Dis Sci. 2013; 58:2682-2690. [PubMed: 23625293]

21. Bonkovsky HL. Hepatotoxicity associated with supplements containing Chinese Green tea (camellia Sinesis). Ann Intern Med. 2006; 144:68-71. [PubMed: 16389263]

22. Kapetanovic IM, Crowell JA, Krishnaraj R, Zakharov A, Lindeblad M, Lyubimov A. Exposure and toxicity of green tea polyphenols in fasted and non-fasted dogs. Toxicology. 2009; 260:28-36. [PubMed: 19464566]

23. Lambert JD, Kennett MJ, Sang S, Reuhl KR, Ju J, Yang CS. Hepatotoxicity of high oral dose (-) epigallocatechin-3-gallate in mice. Food Chem Toxicol. 2010:409-416. [PubMed: 19883714]

24. Daly AK, Day CP. Genetic association studies in drug-induced liver Injury. Sem Liv Dis. 2009; 29:400-411.

25. United States Census Bureau. U.S. Census Bureau; QuickFacts Beta 2010-2014 [Internet]. Available at http://www.census.gov/quickfacts/table/PST045214/00 [Accessed 10/26/2015]

26. Johnston DI, Chang A, Viray M, et al. Hepatotoxicity associated with the dietary supplement OxyELITE Pro- Hawaii, 2013. Drug Test Analysis. 2015

27. Saper RB, Phillips RS, Sehgal A, et al. Lead, mercury, and arsenic in US and Indian manufactured ayurvedic medicines sold via the internet. JAMA. 2008; 300:915-923. [PubMed: 18728265]

28. Martin DJ, Partridge BJ, Shields W. Hepatotoxicity associated with the dietary supplement N.O.xplode. Ann Intern Med. 2013; 159:503. [PubMed: 24081297]

29. Fong TL, Klontz KC, Canas-Coto A, et al. Hepatotoxicity due to hydroxycut: A case series. Am J Gastroenterol. 2010; 105:1561-1566. [PubMed: 20104221]

30. Teschke R, Schulze J, Eickhoff A, Wolff A, Frenzel C. Mysterious Hawaii Liver Disease Case: Naproxen overdose as cause rather than OxyELITE Pro? J Liver Clin Res. 2015; 2(2):1013.

Dig Dis Sci. Author manuscript; available in PMC 2017 September 01. 

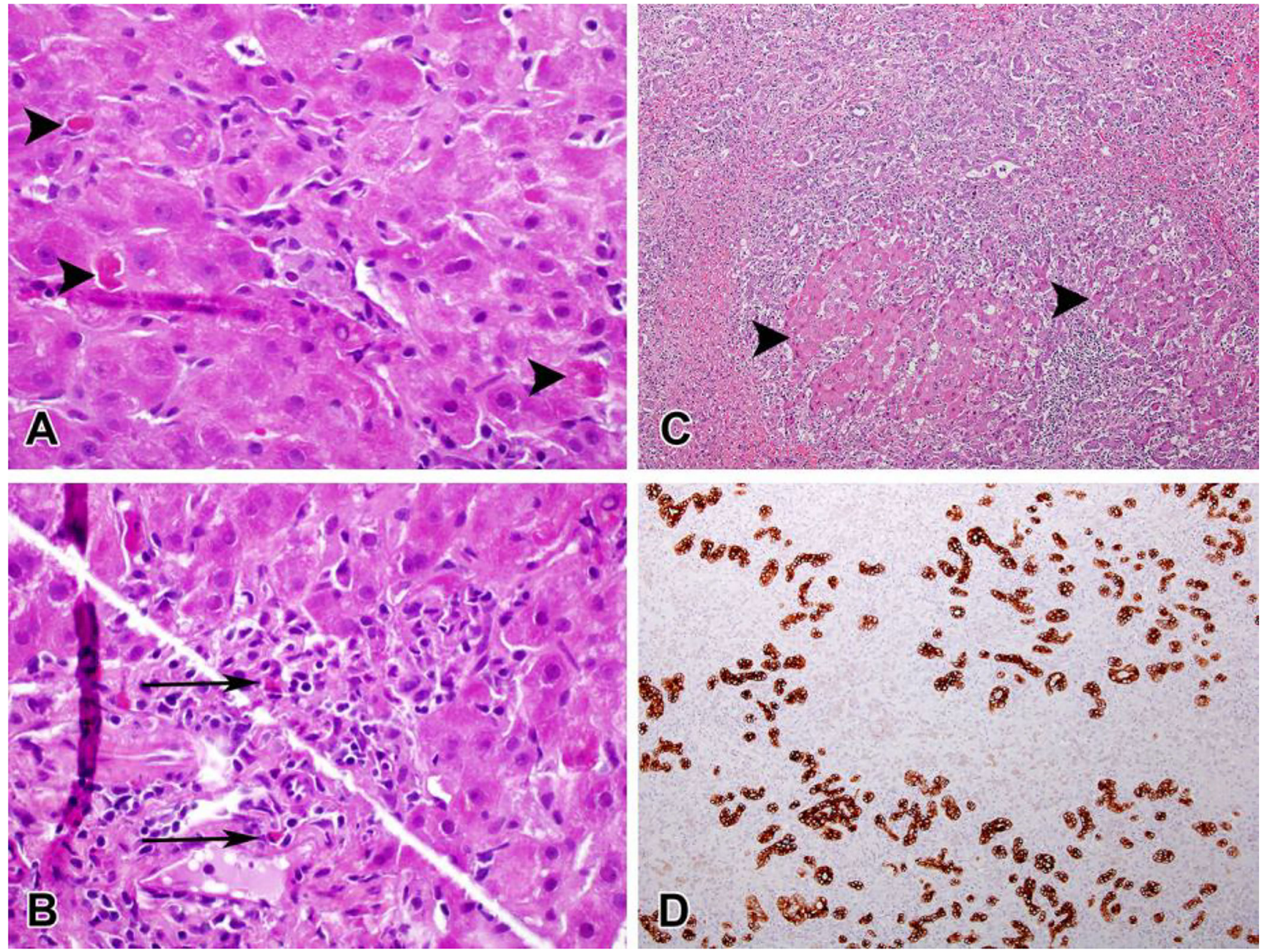

Figure 1.

Two cases of OxyELITE Pro-related liver injury demonstrating mild (A\&B-Case \#3) and severe (C\&D, case \#2) damage. A. Multiple apoptotic hepatocytes are seen in this single high-power field (arrowheads). Foci of macrophages and lymphocytes are present in the center of the field. (H\&E, 600×) B. Portal area showing mild lymphocytic inflammation with interface hepatitis. Several eosinophils are seen (arrows). (H\&E, 600×) C. Severe acute hepatitis with massive parenchymal necrosis. Only a few islands of hepatocytes remain (arrowheads). (H\&E, 100×) D. Staining for keratin 7 shows ductular reaction in the areas of parenchymal loss. (anti-keratin 7 immunostain, 100×). 


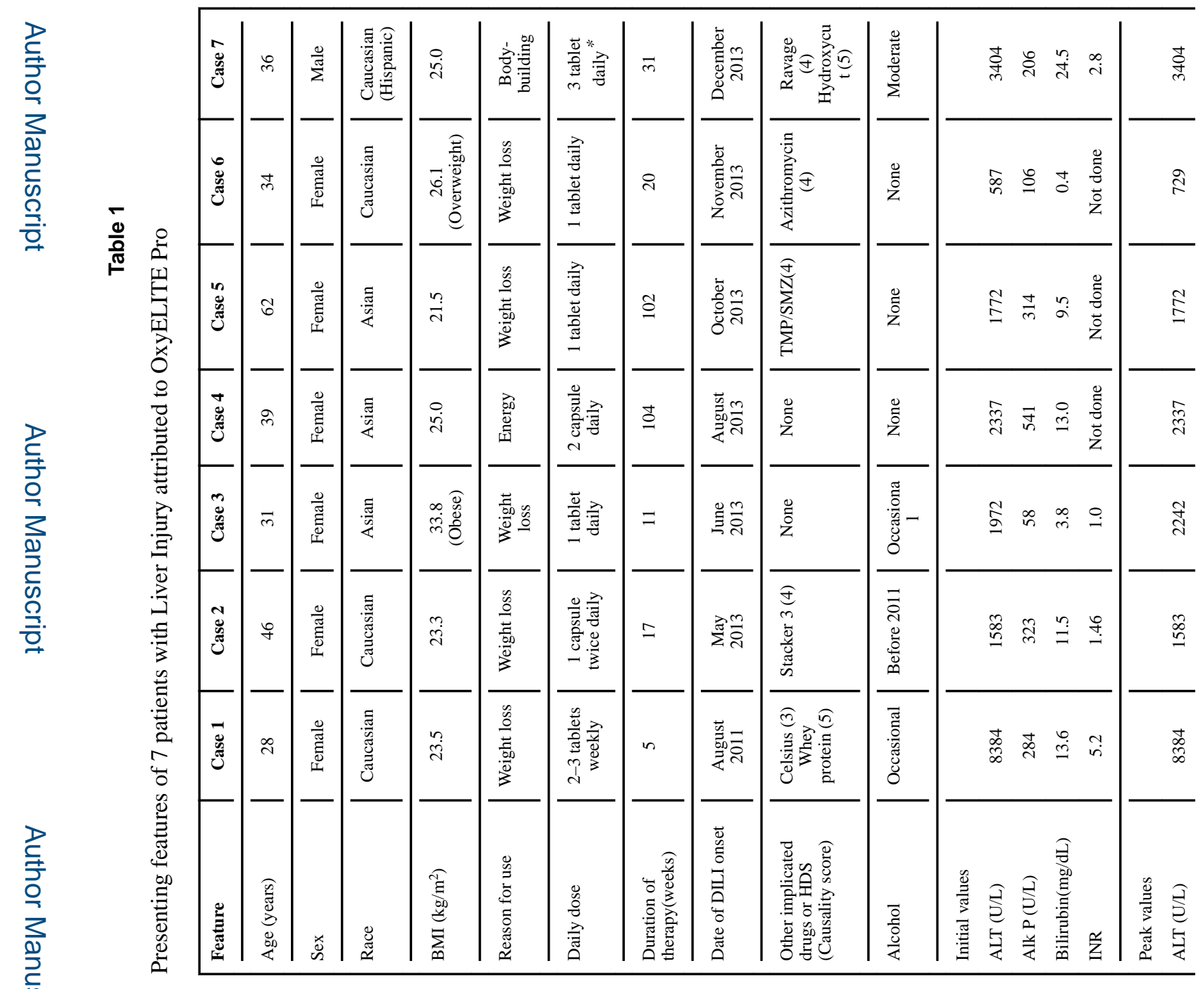




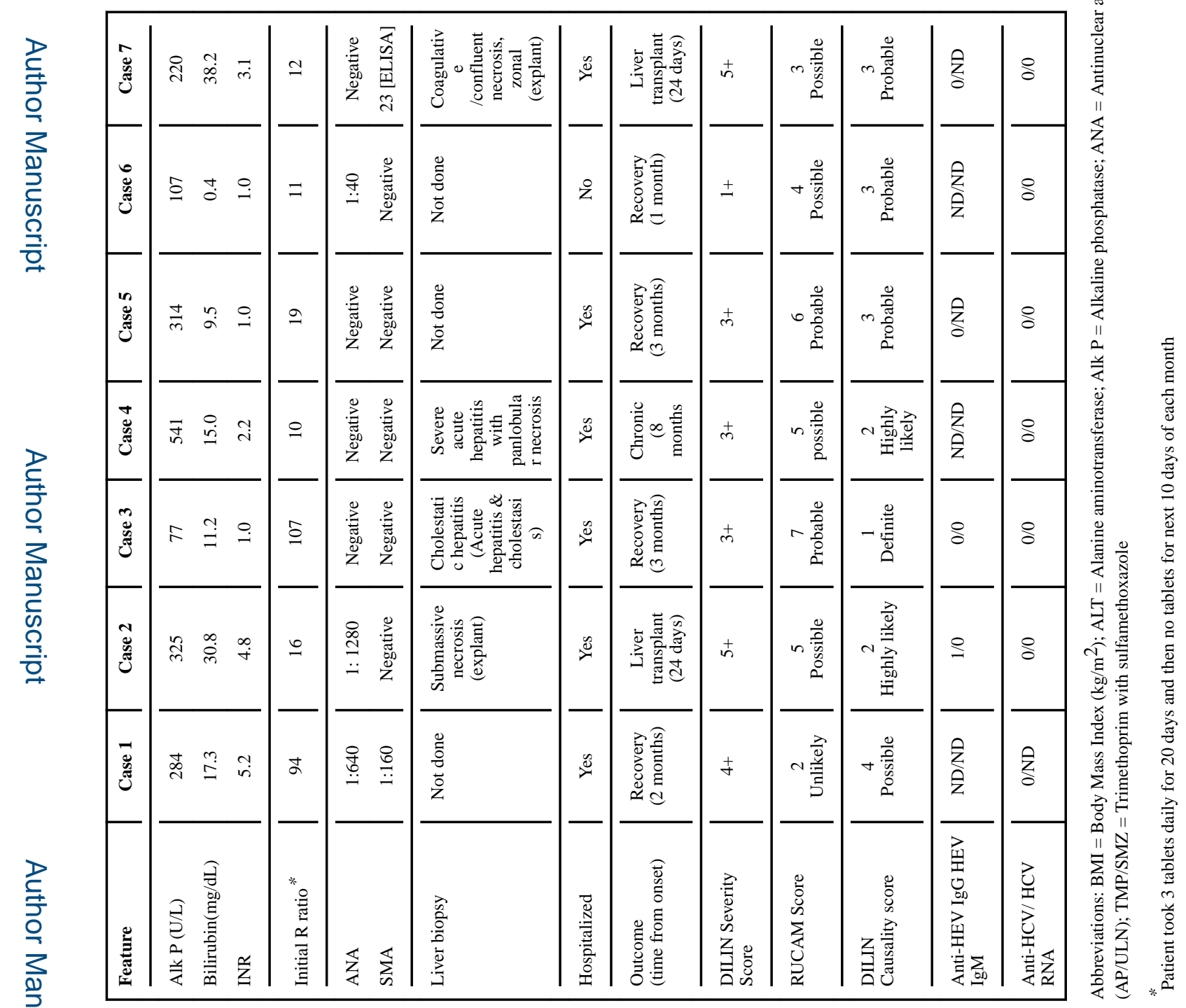


Table 2

Comparison of OxyELITE Pro Hepatotoxicity cases from DILIN and the CDC

\begin{tabular}{|l|c|c|}
\hline & $\begin{array}{c}\text { DILIN Cases } \\
(\mathbf{n = 7})\end{array}$ & $\begin{array}{c}\text { CDC cases } \\
(\mathbf{n = 2 9})\end{array}$ \\
\hline Median (range) age): years & $36(28-62)$ & $33(16-66)$ \\
\hline Time of Onset: Range & Aug 2011-Oct 2013 & May - Oct 2013 \\
\hline $\begin{array}{l}\text { Median (range) duration of OEP use; } \\
\text { days }\end{array}$ & $129(37,711)$ & 60 \\
\hline Female sex (\%) & $6(86 \%)$ & $16(55 \%)$ \\
\hline Asian race (\%) & $3(43 \%)$ & $(>80 \%)$ \\
\hline Median (range) peak ALT: U/L & $2242(729-8,384)$ & $1793(347-3,091)$ \\
\hline Median (range) peak bilirubin: mg/dL & $15(0.4,38.3)$ & $12.6(2.8,39.6)$ \\
\hline Liver biopsy (\%) & $4(57 \%)$ & $10(34 \%)$ \\
\hline Hospitalized (\%) & $6(86 \%)$ & $11(38 \%)$ \\
\hline Liver transplant or death (\%) & $2(29 \%)$ & $3(10 \%)$ \\
\hline Use of concomitant HDS (\%) & $5(71 \%)$ & $12(41 \%)$ \\
\hline
\end{tabular}

ALT $=$ Alanine aminotransferase $(\mathrm{U} / \mathrm{L}) ; \mathrm{HDS}=$ Herbal and Dietary Supplement 